\title{
Die Artenkenntnis von Berliner Schüler_innen am Beispiel einheimischer Vögel
}

\author{
Ulrike Sturm $^{1}$ (D) S Silke Voigt-Heucke ${ }^{1} \cdot$ Kim G. Mortega $^{1} \cdot$ Alexandra Moormann $^{1}$ \\ Eingegangen: 19. November 2019/ Angenommen: 14. August 2020 / Online publiziert: 11. September 2020 \\ (c) Der/die Autor(en) 2020
}

\section{Zusammenfassung}

Der dramatische Rückgang der Biodiversität gehört derzeit zu den größten Problemen weltweit. Das Verständnis, der Erhalt und die Förderung von Biodiversität sind eng verbunden mit Artenkenntnis. Einige Studien zeigten bereits, dass in der Gesellschaft - insbesondere auch bei jungen Menschen - die Kenntnis heimischer Arten gering ist und stetig abnimmt. Es liegen bislang noch keine Daten für Berlin vor. Wir haben daher in einer Querschnittsstudie 186 Schüler_innen der siebten Jahrgangsstufe an drei Schulen in Berlin hinsichtlich ihrer persönlichen Einschätzung der Kenntnis von acht in Berlin häufig vorkommender Vogelarten befragt und anschließend die tatsächlich vorhandene Artenkenntnis geprüft. Die Berliner Schüler_innen erkannten visuell im Mittel zwei Vogelarten und akustisch keine der getesteten Arten. Damit kann die Annahme einer geringen Artenkenntnis in Bezug auf heimische Vogelarten auch für Schüler_innen in Berlin bestätigt werden. Diese Ergebnisse zeigen, dass ein erhöhter Bedarf an der Vermittlung von Artenkenntnis besteht. Dem Erkennen und Beobachten von Tieren und Pflanzen - und damit die Wertschätzung der Vielfalt der Natur - muss mehr Bedeutung zugesprochen werden und geeignete Lernangebote weiterentwickelt und gefördert werden.

Schlüsselwörter Artenkenntnis · Biodiversität · Erwerb von Artenkenntnis

\section{The Species Knowledge of Berlin Students Using the Example of Native Birds}

\begin{abstract}
The dramatic decline in biodiversity is currently one of the greatest problems worldwide. Understanding, preserving and promoting biodiversity is closely linked to species knowledge. Several studies have already shown that in society-especially among young people-knowledge of native species is low and steadily declining. Data for Berlin are not yet available. Therefore, in a cross-sectional study, we interviewed 186 seventh-grade students at three schools in Berlin about their personal assessment of the knowledge of eight bird species frequently occurring in Berlin and then tested the actual knowledge of the species. On average, the Berlin students recognized two bird species visually but none of the tested species acoustically. Thus, the assumption of a low level of species knowledge with regard to native bird species can also be confirmed for students in Berlin. These results show that there is an increased need to communicate and teach species knowledge. The recognition and observation of animals and plants—and thus the appreciation of nature's diversity-must be given more importance and suitable learning opportunities must be further developed and promoted.
\end{abstract}

Keywords Species knowledge $\cdot$ Biodiversity $\cdot$ Acquisition of species knowledge

Zusatzmaterial online Zusätzliche Informationen sind in der Online-Version dieses Artikels (https://doi.org/10.1007/s40573020-00117-8) enthalten.

Ulrike Sturm

Ulrike.Sturm@mfn.berlin

1 Museum für Naturkunde Leibniz-Institut für Evolutions-und Biodiversitätsforschung, Berlin, Deutschland

\section{Einleitung}

Der Verlust von Biodiversität gehört zu den drängenden globalen Problemen unserer Zeit (Steffen et al. 2015; Díaz et al. 2019). Artenkenntnis ist eine Schlüsselqualifikation für ein breites gesellschaftliches Verständnis der globalen Bedeutung von Biodiversität und Naturschutz (Eschenha- 
gen 1985; van Weelie und Wals 2010; Sturm und Berthold 2015). Arten- und Formenkenntnis sind die Basis für das Verständnis des Lebendigen und der Vielfalt (Eschenhagen 1985), das Engagement für Natur (Sturm und Berthold 2015) und den täglichen Umgang mit Natur, z. B. (Mayer und Horn 1993). Zudem wirkt sich insbesondere in Städten die empfundene Artenvielfalt positiv auf das Wohlbefinden der dort lebenden Menschen aus (Dallimer et al. 2012; Cox und Gaston 2015). Die Kenntnis über die Natur, die Menschen im Alltag begegnet, und ihrer Veränderung ist eine wichtige Grundlage für die Kompetenz globale Zusammenhänge zu verstehen und einen Beitrag zu den Nachhaltigkeitszielen der UN zu leisten (Rieckmann et al. 2017). Gleichzeitig ist Artenkenntnis eine wichtige Wissensgrundlage für Forschung und Naturschutz. Das Vorhandensein von Artenkenntnis und taxonomischen Wissens ermöglicht die Kommunikation über Arten sowie die Dokumentation und Untersuchung von Artenvielfalt (Wägele et al. 2011; Schulte et al. 2019).

\section{Der Begriff Artenkenntnis}

Artenkenntnis wird häufig synonym mit dem Begriff der Formenkenntnis verwendet und oft fehlt eine klare Definition der Terminologie. Mayer und Horn (1993) prägten ein erweitertes Verständnis von Formenkenntnis, das neben der reinen Zuordnung des Namens einer Art auch die Kenntnis des taxonomischen sowie des ökologischen, kulturellen und gesellschaftlichen Kontexts mit einbezieht. Hooykaas et al. (2019) führten das Konzept der Species Literacy ein. Sie umfasst Faktenwissen und grundlegendes Bewusstsein und Verständnis von Arten, aber auch Kompetenzen und Fertigkeiten wie die Beobachtung von Arten und die Anwendung von Wissen. In der Taxonomie werden Lebewesen erforscht, benannt, klassifiziert und in einer hierarchischen Einteilung in Gruppen eingeteilt (Tancoigne et al. 2011). Wissenschaftler_innen geben Taxa eine eindeutige Entsprechung, die verwendet wird, um weitere Individuen zu identifizieren (Wägele et al. 2011).

In unserer Studie definieren wir Artenkenntnis, in Abgrenzung zur erweiterten Formenkenntnis von Mayer und Horn (1993) und Taxonomie, basierend auf dem Konzept der Species Literacy als die grundlegende Fähigkeit Arten zu erkennen. Die Fähigkeit Arten zu erkennen geht über die reine Kenntnis von Namen hinaus und setzt sich aus drei zentralen Komponenten zusammen: artspezifische Merkmale erkennen, Arten benennen und der damit verbundenen Fähigkeit, Arten zu unterscheiden. Artenkenntnis umfasst in diesem Sinne ein grundlegendes System von Wissen, Fähigkeiten und Fertigkeiten, die für eine erweiterte Formenkenntnis und taxonomisches Wissen benötigt werden.
Artenkenntnis kann unterschiedlich ausgeprägt sein, da das Erkennen von artspezifischen Merkmalen für unterschiedliche Ordnungen, Familien, Großgruppen und sogar Gattungen teilweise sehr unterschiedliche Kenntnisse und Qualifikationen erfordert. Schulte et al. (2019) beschreiben den Erwerb von Artenkenntnis als handlungsorientierten Selbstlernprozess, der in unterschiedlichen Qualifizierungsstufen erfolgt und meist der Unterstützung von Mentor_innen bedarf. Beispielsweise können viele Insektenarten auf Artebene nur durch eine Präparation getöteter Tiere bestimmt werden. Auch werden Schwierigkeiten von Anfänger_innen für die Bestimmung von Pflanzen (Hesse 2002; Jäkel und Schaer 2004; Buck et al. 2019) und von Vögeln anhand akustischer Merkmale (Bergmann 2017) beschrieben.

\section{Wissensstand zur Artenkenntnis von Schüler_innen in Deutschland}

Seit Jahrzehnten wird beklagt, dass die Artenkenntnis in der Gesellschaft verloren geht. Immer wieder wird gemahnt, dass Jugendlichen in Deutschland oft konkretes Wissen über die Natur fehlt (Brämer et al. 2016) und die Zahl der Artenkenner_innen abnimmt (Frobel und Schlumprecht 2016). Die Schule wurde für den Erwerb von Artenkenntnis immer wieder als wichtiger Lernort erwähnt (Frobel und Schlumprecht 2016) und die Kultusministerkonferenz forderte bereits im Jahr 2004, dass Artenkenntnis in allen Schularten verbindlich vermittelt werden muss (KMK 2004). Nach Mayer (1992) herrscht seit über 100 Jahren eine mangelnde Arten- bzw. Formenkenntnis von Schüler_innen in Deutschland. Diese Aussage bisher jedoch nicht empirisch überprüft.

Einheimische Vögel sind ein häufiges Studienobjekt in der Untersuchung von Artenkenntnis, da sie meist gut beobachtbar und ohne Hilfsmittel anhand einfacher Merkmale bestimmbar sind (Zahner et al. 2007; Randler und Wieland 2010). Dadurch liegen einige Untersuchungsergebnisse zu Artenkenntnissen von Vogelarten bei Schüler_innen vor (Tab. 1). Unterschiedliche Voraussetzungen bedingt durch das föderale Schulsystem in Deutschland und teilweise lokal oder regional unterschiedliches Vorkommen von Arten erschweren die Vergleichbarkeit und Übertragung der Ergebnisse, die bislang empirische Einblicke in die Artenkenntnis von Schüler_innen am Beispiel einheimischer Tierarten in Baden-Württemberg (Randler 2003, 2006, 2008a), Bayern (Zahner et al. 2007; Gerl et al. 2018), Schleswig-Holstein (Randler 2006, 2008a) und Niedersachsen (Randler 2006, 2008a) geben.

In den letzten 20 Jahren dokumentierten dennoch eine Reihe von Studien Trends im zeitlichen Vergleich der Artenkenntnis von Schüler_innen (Randler 2006, 2008a; Zahner et al. 2007; Gerl et al. 2018). Randler (2006, 2008a) 
Tab. 1 Übersicht empirischer Studien über Arten- und Formenkenntnis von Vogelarten bei Schüler_innen in Deutschland seit 2000

\begin{tabular}{lll}
\hline Autor_innen & Untersuchungsgegenstand & Personenkohorte \\
\hline $\begin{array}{l}\text { Randler und Bogner } \\
(2002)\end{array}$ & $\begin{array}{l}\text { Methoden zur Förderung von Ar- } \\
\text { tenkenntnis am Beispiel Vögel }\end{array}$ & Jahrgangsstufe 5 und 6 $(N=242)$ \\
Randler (2003) & Artenkenntnis am Beispiel Vögel & Jahrgangsstufe 5-8 $(N=134)$ in Baden-Württemberg \\
Randler (2006), & $\begin{array}{l}\text { Artenkenntnis am Beispiel Wirbel- } \\
\text { tiere }\end{array}$ & $\begin{array}{l}\text { Alter: 9-19 Jahre }(N=879) \text {, in Schleswig-Holstein }(N=139) \text {, Niedersachsen } \\
(N=222) \text { und Baden-Württemberg }(N=518) \text { und Jahrgangsstufe 4 }(N=65)\end{array}$ \\
$\begin{array}{ll}\text { Schreck und Randler } \\
(2007)\end{array}$ & $\begin{array}{l}\text { Unterrichtskonzepte zur Vermitt- } \\
\text { lung von Vogelstimmen }\end{array}$ & Jahrgangsstufe 5 und 6 $(N=96)$ \\
Zahner et al. (2007) & Artenkenntnis am Beispiel Vögel & Jahrgangsstufe 4, 7 und 12 $(N=3228)$ in Bayern \\
Gerl et al. (2018) & Artenkenntnis am Beispiel Vögel & Alter: 6-19 Jahre $(N=1957)$ in Bayern \\
\hline
\end{tabular}

stellte in einem Vergleich der Artenkenntnis von Wirbeltieren bei Schüler_innen zwischen den Jahren 1981 und 2005 keine signifikante Abnahme der Artenkenntnis fest. Zahner und Kolleg_innen (2007) fanden jedoch am Beispiel einheimischer Brutvögel einen Rückgang der Artenkenntnis im Vergleich zu der Studie von Eschenhagen aus dem Jahr 1981. Gerl und Kolleg_innen bestätigten einen Rückgang der Fähigkeiten der Schüler_innen in Bayern einheimische Vogelarten korrekt zu benennen. Jedoch zeigten die Ergebnisse ebenfalls Indizien für eine Zunahme der Bekanntheit für vier von fünf Vogelarten.

Die größte Artenkenntnis bei Schüler_innen wurde in der Forschungsliteratur für 14- bis 15-Jährige dokumentiert (Gerl et al. 2018) und scheint von der 7. Jahrgangsstufe bis zur 12. Jahrgangsstufe nicht mehr bedeutend anzusteigen (Randler 2003). Einige Studien fanden im Vergleich der Schulformen Unterschiede in der Artenkenntnis der Schüler_innen (Randler 2006), andere konnten wiederum keinen Effekt aufzeigen (Gerl et al. 2018). Neben der Schule scheinen die Eltern der wichtigste Einfluss auf die persönlichen Naturbeobachtungen und auf die Artenkenntnis zu sein (Gerl et al. 2018; Remmele und Lindemann-Matthies 2018).

Obwohl Vogelstimmen unter Ornithologen ein wichtiges Bestimmungsmerkmal sind, wurde bei Schüler_innen das Erkennen von Vogelarten anhand akustischer Merkmale bisher kaum untersucht. Schreck und Randler (2007) stellten jedoch in ihrer Studie zu Unterrichtskonzepten zur Vermittlung von Vogelstimmen eine geringe Kenntnis von Vogelstimmen und einen sehr schwachen Lernzuwachs fest.

Befragungen zur Artenkenntnis basieren teilweise auf der reinen Selbsteinschätzung der Schüler_innen. Zur Zuverlässigkeit der Selbsteinschätzung der Artenkenntnis von Schüler_innen gibt es jedoch widersprüchliche Ergebnisse. Hesse (2002) fand beispielsweise eine lediglich geringe Übereinstimmung zwischen der Selbsteinschätzung und der tatsächlichen Artenkenntnis bei Frühblühern. Dem entgegen zeigten Jäkel und Schaer (2004), dass die Schüler_innen zutreffende Voraussagen bezüglich der Artenkenntnis von Pflanzen machten.

\section{Ziel der Studie und Forschungsfragen}

Bisher liegen in der Forschungsliteratur zur Artenkenntnis von Schüler_innen aus Berlin - in Bezug auf ihre Selbsteinschätzung und die tatsächliche Kenntnis über Arten - keine Daten vor. Das Ziel unseres Beitrags ist es, diese Lücke zu schließen und eine Grundlage für die Untersuchung von zeitlichen Trends sowie für die Entwicklung von Lernangeboten zu schaffen. Wir untersuchen daher die visuelle und akustische Artenkenntnis von Schüler_innen am Beispiel der anzunehmenden höchsten Kenntnis (Randler 2006; Gerl et al. 2018) der in Berlin häufig vorkommender Brutvogelarten. Aus diesem Forschungsvorhaben leiteten sich folgende Fragen ab:

F1 Wie schätzen die befragten Berliner Schüler_innen (Jahrgangsstufe 7) ihre eigene Artenkenntnis am Beispiel einheimischer Vogelarten ein? Inwiefern besteht in der Selbsteinschätzung ein Unterschied zwischen akustischer und visueller Artenkenntnis?

F2 Welche Artenkenntnis über einheimische Vögel haben Berliner Schüler_innen (Jahrgangsstufe 7)? Inwiefern besteht ein Unterschied zwischen akustischer und visueller Erkennung?

F3 Inwiefern besteht ein Unterschied in der Selbsteinschätzung und in der tatsächlich gemessenen Artenkenntnis der befragten Berliner Schüler_innen (Jahrgangsstufe 7)?

Nach dem Modell der Didaktischen Rekonstruktion sind die Schülervorstellungen ein wichtiger Bestandteil für die Entwicklung von Lernangeboten (Kattmann et al. 1997). Studien zeigen, dass Schüler_innen Arten nach unterschiedlichen Kriterien ordnen und die Methode des Vergleichens nicht beherrschen (Krüger und Burmester 2005). Eine Analyse von falschen Benennungen können Anhaltspunkte geben, um die von den Schüler_innen angewandten Entscheidungen und Kriterien bei der Artbestimmung besser zu verstehen (Hesse 2002). 
F4 Inwieweit geben die von den befragten Berliner Schüler_innen (Jahrgangsstufe 7) gemachten Verwechslungen Hinweise auf die Kriterien, die sie bei der Zuordnung verwendeten?

\section{Methoden}

\section{Stichprobe}

An der Studie nahmen insgesamt 186 Schüler_innen der 7. Jahrgangsstufe in elf Klassen von drei Schulen unterschiedlichen Schultyps in Berlin teil. Nach den Vorgaben des Berliners Rahmenlehrplan sollten alle Berliner Schüler_innen in der 7. Klasse eine schulische Grundbildung im Bereich der Artenkenntnis haben (Senatsverwaltung für Bildung Jugend und Sport Berlin 2006). 96 (52\%) der teilnehmenden Schüler_innen besuchten ein Gymnasium, 67 (36\%) der befragten Schüler_innen eine Sekundarschule und 23 (12\%) Schüler_innen eine Gemeinschaftsschule. $77(41 \%)$ der teilnehmenden Schüler_innen identifizierten sich als weiblich, 68 (37\%) Schüler_innen identifizierten sich als männlich, ein_e $(0,5 \%)$ Schüler_in identifizierte sich als divers und $40(21,5 \%)$ Schüler_innen machten kei- ne Angaben zu ihrem Geschlecht. An der vorausgehenden Befragung zur Selbsteinschätzung nahmen 149 (80\% der Gesamtstichprobe) Schüler_innen teil.

\section{Studiendesign}

Die Querschnittsstudie mit zwei Phasen (Phase 1: Selbsteinschätzung der Artenkenntnis, Phase 2: tatsächliche Artenkenntnis) wurde von Dezember 2018 bis Januar 2019 in Berlin durchgeführt. In der ersten Phase erhielten die Schüler_innen im Regelunterricht von der jeweiligen Lehrkraft einen Fragebogen zur Selbsteinschätzung ihrer Artenkenntnis anhand des deutschen Artnamens von 16 einheimischen Brutvogelarten. In der zweiten Phase wurde die Artenkenntnis derselben Schüler_innen für acht Brutvogelarten geprüft (Tab. 2). Es wurde eine Auswahl aus der ersten Phase getestet, um die Möglichkeit einer zufällig korrekten Zuordnung der in der ersten Phase in dem Fragebogen vorgegebenen Artnamen nach dem Ausschlussverfahren zu reduzieren. Jede_r Schüler_in wurde einzeln an zwei Stationen (visuelle und akustische Erkennung) in einem standardisierten Ablauf von geschulten Personen getestet. Für jede Vogelart spielten die Befragenden den Schüler_innen jeweils für fünf Sekunden eine Videoaufnahme ohne Ton (visuelle Erken-

Tab. 2 Auswahl der acht getesteten Vogelarten, Häufigkeit= Status und Bestand (Witt und Steiof 2013), Bestimmungsmerkmale ơ nach Svensson et al. (1999)

\begin{tabular}{|c|c|c|c|}
\hline$\overline{\text { Art }}$ & $\begin{array}{l}\text { Häufigkeit } \\
\text { (RL Berlin) }\end{array}$ & Wichtige Bestimmungsmerkmale $\sigma^{7}$ & $\begin{array}{l}\text { Mögliche Verwechslungsarten } \sigma^{7} \\
(A=\text { Aussehen, } S=\text { Stimme) }\end{array}$ \\
\hline $\begin{array}{l}\text { Amsel (Turdus } \\
\text { merula) }\end{array}$ & $\begin{array}{l}\text { Regelmäßiger } \\
\text { Brutvogel: } \\
34.000-74.000\end{array}$ & $\begin{array}{l}\text { Gefiederfarbe, Schnabelfarbe, Farbe des Lidrings, Schwanz- } \\
\text { form; Stimme: melodisch und weich, klar und laut, gemächli- } \\
\text { ches Tempo, oft große Tonsprünge }\end{array}$ & Dohle (A), Misteldrossel (S), Star (A) \\
\hline $\begin{array}{l}\text { Buchfink } \\
\text { (Fringilla } \\
\text { coelebs) }\end{array}$ & $\begin{array}{l}\text { Regelmäßiger } \\
\text { Brutvogel: } \\
4900-5700\end{array}$ & $\begin{array}{l}\text { Gefiederfarbe; Stimme: kraftvoll schmetternde Strophe, Ende } \\
\text { mit einem kleinen Schnörkel }\end{array}$ & Bergfink (A), Rotkehlchen (A) \\
\hline $\begin{array}{l}\text { Hausrotschwanz } \\
\text { (Phoenicurus } \\
\text { ochruros) }\end{array}$ & $\begin{array}{l}\text { Regelmäßiger } \\
\text { Brutvogel: } \\
4000-6000\end{array}$ & $\begin{array}{l}\text { Gefiederfarbe, insbesondere Schwanz; Stimme: knarrender } \\
\text { Gesang }\end{array}$ & Gartenrotschwanz (A) \\
\hline $\begin{array}{l}\text { Haussperling } \\
\text { (Passer dome- } \\
\text { sticus) }\end{array}$ & $\begin{array}{l}\text { Regelmäßiger } \\
\text { Brutvogel: } \\
\text { 110.000-130.000 }\end{array}$ & $\begin{array}{l}\text { Gefiederfarbe, insbesondere Scheitel, Wangen und Hals; Stim- } \\
\text { me: charakteristischer Tschilp-Tschilp Gesang }\end{array}$ & $\begin{array}{l}\text { Feldsperling (A, S), Heckenbraunel- } \\
\text { le (A) }\end{array}$ \\
\hline $\begin{array}{l}\text { Kohlmeise } \\
\text { (Parus major) }\end{array}$ & $\begin{array}{l}\text { Regelmäßiger } \\
\text { Brutvogel: } \\
28.000-55.000\end{array}$ & $\begin{array}{l}\text { Gefiederfarbe und -zeichnung, insbesondere der Unterseite und } \\
\text { Flügel; Stimme: „zizibäh zizibäh“ Ruf }\end{array}$ & Blaumeise (A), Tannenmeise (A) \\
\hline $\begin{array}{l}\text { Nachtigall } \\
\text { (Luscinia me- } \\
\text { garhynchos) }\end{array}$ & $\begin{array}{l}\text { Regelmäßiger } \\
\text { Brutvogel: } \\
1300-1700\end{array}$ & $\begin{array}{l}\text { Gefiederfarbe; Stimme: sehr kräftiger, melodischer und ab- } \\
\text { wechslungsreicher Gesang, trillernde, flötende und hart } \\
\text { schmetternde Abschnitte }\end{array}$ & $\begin{array}{l}\text { Amsel (S), Gartengrasmücke (A), } \\
\text { Gartenrotschwanz (A), Mönchs- } \\
\text { grasmücke (S), Rotkehlchen (S), } \\
\text { Singdrossel (S), Sprosser (A, S) }\end{array}$ \\
\hline $\begin{array}{l}\text { Rotkehlchen } \\
\text { (Erithacus } \\
\text { rubecula) }\end{array}$ & $\begin{array}{l}\text { Regelmäßiger } \\
\text { Brutvogel: } \\
4400-6600\end{array}$ & $\begin{array}{l}\text { Gefiederfarbe, insbesondere von Gesicht, Kehle und Brust; } \\
\text { Stimme: Gesang beginnt oft mit feinen, hohen Tönen, dann } \\
\text { abfallend, schneller und lauter, abwechselndes Tempo und } \\
\text { veränderter Tonhöhe in perlenden, klaren Tonreihen }\end{array}$ & Buchfink (A), Nachtigall (S) \\
\hline $\begin{array}{l}\text { Star (Sturnus } \\
\text { vulgaris) }\end{array}$ & $\begin{array}{l}\text { Regelmäßiger } \\
\text { Brutvogel: } \\
16.000-32.000\end{array}$ & $\begin{array}{l}\text { Gefiederfarbe, Schwanzform, Schnabelform; Stimme: laute, } \\
\text { langgezogene Pfeiftöne und recht leise knackende, klicken- } \\
\text { de und knirschende Laute, Vielzahl von Imitationen anderer } \\
\text { Vögel oder Geräusche }\end{array}$ & $\begin{array}{l}\text { Amsel (A), Dohle (A), imitiert Ge- } \\
\text { sang beispielsweise von Kiebitz (S), } \\
\text { Brachvogel (S), Haussperling (S), } \\
\text { Elster (S) }\end{array}$ \\
\hline
\end{tabular}


nung) bzw. ein Audio einer Gesangsaufnahme (akustische Erkennung) eines Vogelmännchens vor. Die Schüler_innen wurden bei beiden Stationen gebeten, nach dem Abspielen einer Aufnahme in einem Fragebogen die von ihnen erkannte Vogelart mit dem deutschen Artnamen zu benennen.

Es wurden mit Ausnahme des Geschlechts keine demographischen Daten abgefragt. Die Wiedererkennung der Befragten und Zuordnung der Selbsteinschätzung erfolgte über eine Pseudonymisierung in Form einer sogenannten Identifikationsnummer (Tiemann und Körbs 2014).

Die Befragung wurde durch die Berliner Senatsverwaltung genehmigt. Die Eltern der Schüler_innen erhielten ein Merkblatt, in dem die Details der Studie erläutert wurden und es wurde eine schriftliche Zustimmung von ihnen eingeholt. Die Teilnahme war selbstverständlich freiwillig.

\section{Auswahl der Arten}

Die Auswahl der Arten erfolgte nach den folgenden Kriterien: Häufigkeit in Berlin, Vergleichbarkeit mit anderen Studien, charakteristische visuelle oder akustische Bestimmungsmerkmale.

Es wurden folgende 16 einheimische Brutvogelarten für die Selbsteinschätzung der Artenkenntnis ausgewählt: Amsel (Turdus merula), Blaumeise (Cyanistes caeruleus), Buchfink (Fringilla coelebs), Buntspecht (Dendrocopos major), Grünfink (Chloris chloris), Hausrotschwanz (Phoenicurus ochruros), Haussperling (Passer domesticus), Kohlmeise (Parus major), Mauersegler (Apus apus), Nachtigall (Luscinia megarhynchos), Nebelkrähe (Corvus cornix), Rotkehlchen (Erithacus rubecula), Straßentaube (Columbia livia $f$. urbana), Star (Sturnus vulgaris), Stockente (Anas platyrhynchos), Zilpzalp (Phylloscopus collybita). Der Haussperling wurde zum besseren Verständnis der Schüler_innen auch als Spatz bezeichnet.

Aus diesen Arten wurden acht häufig in Berlin anzutreffende Vogelarten für die Überprüfung der tatsächlichen Artenkenntnis ausgewählt (Tab. 2).

\section{Messinstrumente}

Für die Selbsteinschätzung der Artenkenntnis (1. Phase) und den Test der tatsächlichen akustischen und visuellen Artenkenntnis (2. Phase) entwickelten wir jeweils einen Fragebogen. Der Fragebogen für die Phase 1 fragte die Kenntnis von 16 Vogelarten anhand des Artnamens mit vier Antwortkategorien ab. Zu jedem der 16 Artnamenkonnten die Schüler_innen zwischen folgenden Antworten wählen: „Diesen Vogel erkenne ich, wenn ich ihn sehe.“, „Diesen Vogel erkenne ich, wenn ich ihn höre.“, „Diesen Vogel kann ich nicht erkennen. Ich habe den Namen aber schon einmal gehört.“, ,,Von diesem Vogel habe ich noch nie gehört.“. Die
Schüler_innen konnten mehr als eine Antwortmöglichkeit pro Vogelart auswählen.

Der Fragebogen für die Phase 2 testete die tatsächliche visuelle und akustische Artenkenntnis der Schüler_innen für acht Vogelarten (Tab. 2). Der Fragebogen fragte den deutschen Artnamen zu je einer Video- oder Tonaufnahme ab. Alternativ konnten die Schüler_innen die folgenden Antwortmöglichkeiten wählen: „Diesen Vogel habe ich schon einmal gehört, bzw. gesehen, aber ich kenne den $\mathrm{Na}$ men nicht.“, „Kenne ich nicht.“.

Die acht ausgewählten Vogelarten wurden für die visuelle Bestimmung anhand von Videos und für die akustische Bestimmung mit Audioaufnahmen über Kopfhörer randomisiert präsentiert. Wir wählten Videoaufnahmen aus, die alle für eine zuverlässige Bestimmung relevanten Merkmale zeigten. Alle Videos wurden von der Videoplattform YouTube entnommen und von uns auf eine Dauer von fünf Sekunden geschnitten. Diese Dauer gewährleistete, dass jeder Vogel in seinem natürlichen Verhalten gezeigt werden konnte. Wir stellten eine vergleichbare Darstellung der Vogelarten in den Videos sicher. So zeigten wir lediglich Männchen und starteten immer mit einem schwarzen Bildschirm (1s). Zum Testen der akustischen Artenkenntnis stellten wir Gesangsaufnahmen, die besonders charakteristische Merkmale des Gesangs wiedergeben, aus dem Tierstimmenarchiv des Museum für Naturkunde Berlin sowie von der Plattform Xeno Canto zusammen. Jede Gesangsaufnahme schnitten wir auf fünf Sekunden und starteten immer mit einer kurzen Zeitverzögerung (1s; vergleichbar mit dem schwarzen Bildschirm). Die Schüler_innen notierten den Namen der Art jeweils in dem Fragebogen oder wählten eine der alternativen Antwortmöglichkeiten. Wenn ein_e Schüler_in den Wunsch äußerte, die visuellen und akustischen Aufnahmen noch einmal sehen/hören zu dürfen, wurden die Aufnahmen ein weiteres Mal abgespielt.

Die Antworten wurden im Rahmen eines Punktesystems ausgewertet. Für jeden korrekten Artnamen wurde ein Punkt und für eine korrekt zugeordnete Gattung ein halber Punkt vergeben (Gerl et al. 2018). In der ornithologischen Praxis gibt es unterschiedliche Ansichten zur Bewertung der Nutzung der Bezeichnung Spatz. Wir werteten die Bezeichnung Spatz für den Haussperling als Artname und vergleichend als Gattungsbezeichnung aus. Für die Selbsteinschätzung wurden die Antworten „Diesen Vogel erkenne ich, wenn ich ihn sehe.“ und „Diesen Vogel erkenne ich, wenn ich ihn höre." jeweils mit einem Punkt bewertet. Die Antworten „Diesen Vogel kann ich nicht erkennen. Ich habe den Namen aber schon einmal gehört." und „Von diesem Vogel habe ich noch nie gehört.“ wurden als ,nicht erkennen“"vereint und null Punkte vergeben. 
Abb. 1 Prozentualer Anteil der Selbsteinschätzung der befragten Schüler_innen zu ihrer Artenkenntnis (visuell und/oder akustisch) für 16 Vogelarten, $N=149$. Ein hoher prozentualer Anteil bedeutet, dass viele Schüler_innen in der Phase 1 der Studie angaben, eine Vogelart anhand von akustischer Merkmale, anhand akustischer und visueller, oder visueller Merkmale zu erkennen

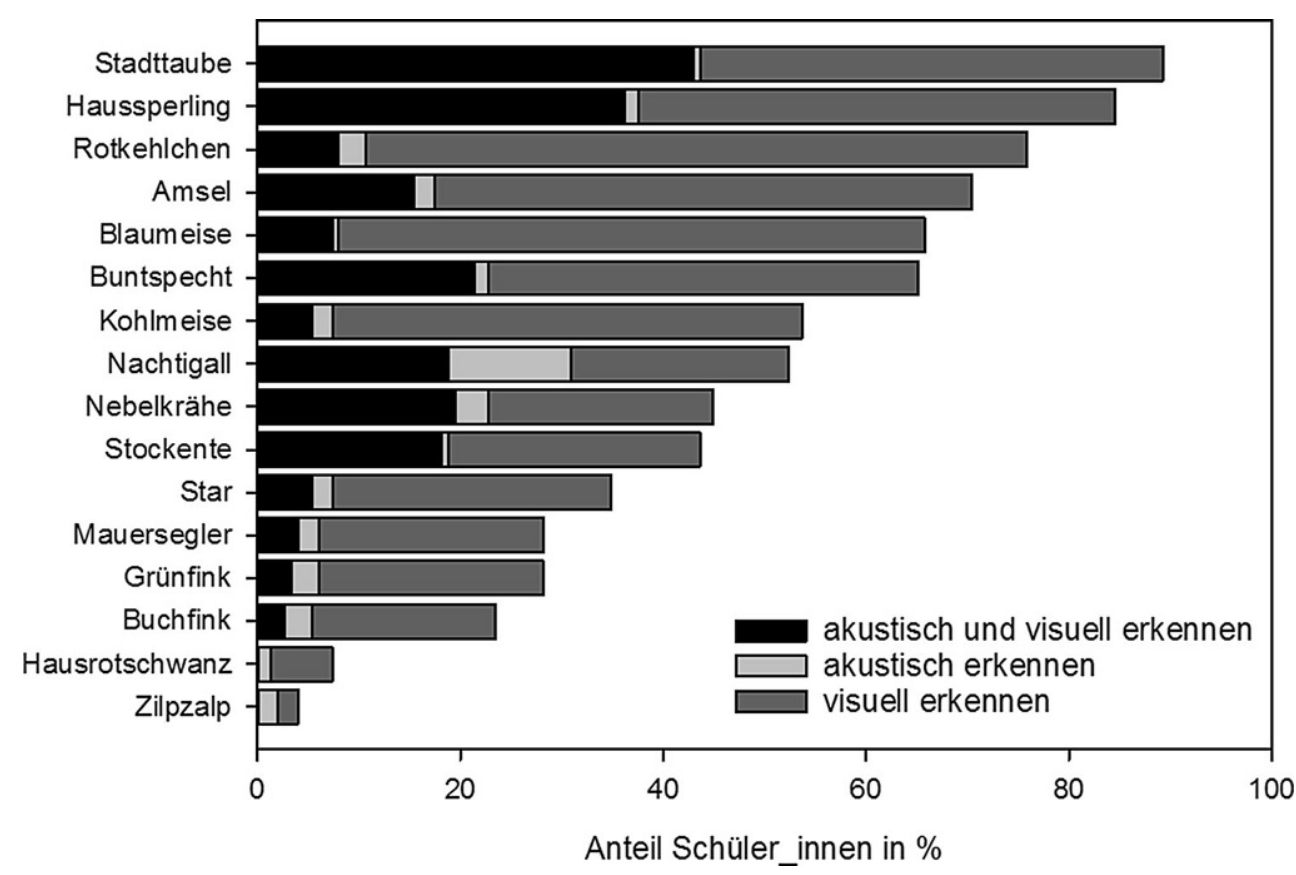

\section{Datenanalyse}

Alle 146 Fragebögen aus der Phase 1 zur Selbsteinschätzung und alle 186 Fragebögen des Tests der tatsächlichen Artenkenntnis aus der Phase 2 wurden ausgewertet. Bei fehlenden oder widersprüchlichen Antworten der Selbsteinschätzung (z. B. gleichzeitige Auswahl der Antwort „Diesen Vogel erkenne ich, wenn ich ihn höre" und „Von diesem Vogel habe ich noch nie gehört.") wurde die jeweilige Art nicht berücksichtigt. Es wurden von 47 Schüler_innen Selbsteinschätzungen ausgeschlossen (32-mal keine Antwort, 76-mal Widerspruch). Widersprüchliche Aussagen kamen bei allen 16 Vogelarten vor.

Angelehnt an Gerl et al. (2018) definierten wir die Punktzahl von mehr als 6,5 Punkten als besonders hohe Artenkenntnisse $\left(\mathrm{h}_{\mathrm{o}}\right)$ und die Punktzahl unter 1,5 Punkten als besonders geringe Artenkenntnis $\left(h_{u}\right)$ und ermittelten jeweils ihren Anteil. Für die Auswertung der Selbsteinschätzung definierten wir eine Punktzahl von mehr als 13 Punkten als besonders hohe Artenkenntnisse $\left(\mathrm{h}_{\mathrm{o}}\right)$ und die Punktzahl unter 3,5 Punkten als besonders geringe Artenkenntnis $\left(h_{u}\right)$. Alle Verwechslungen, die zweimal oder häufiger vorkamen, wurden auf mögliche Gründe der Verwechslung untersucht und geclustert.

Die Daten wurden mit SPSS (Version 26) analysiert. Da jeweils eine schiefe Verteilung vorlag, wurden die Ergebnisse der Selbsteinschätzung und der tatsächlichen Artenkenntnis mit einem Wilcoxon-Test für nichtparametrische, verbundene Stichproben analysiert. Die Effektstärke wurde jeweils mit dem Korrelationskoeffizient $r$ berechnet (Maher et al. 2013).

Tab. 3 Mittlere Anzahl der Vogelarten, die die Schüler_innen nach eigenen Angaben visuell und/oder akustisch erkennen (Median, Variationsbreite), Anteil der Schüler_innen mit geringer Artenkenntnis $\left(\mathrm{h}_{\mathrm{u}}\right)$ und einer hohen Artenkenntnis $\left(\mathrm{h}_{\mathrm{o}}\right), N=149$

\begin{tabular}{llllll}
\hline & $N$ & Median $(\mathrm{Md})$ & Variationsbreite $(\mathrm{R})$ & $\mathrm{H}_{\mathrm{u}}(\%)(<3,5$ Punkte $)$ & $\mathrm{H}_{\mathrm{o}}(\%)(>12,5$ Punkte $)$ \\
\hline Visuell erkennen & 149 & 8 & $15_{\max }-0_{\min }$ & 16 & 8 \\
Akustisch erkennen & 149 & 2 & $11_{\max }-0_{\min }$ & 66 & 0 \\
\hline
\end{tabular}

Tab. 4 Mittlere Testleistung (Median) mit Variationsbreite (R), prozentualer Anteil der Schüler_innen mit geringer Artenkenntnis $\left(\mathrm{h}_{\mathrm{u}}\right) \mathrm{und}$ einer hohen Artenkenntnis $\left(\mathrm{h}_{\mathrm{o}}\right), N=186$

\begin{tabular}{llllll}
\hline & $N$ & Median $(\mathrm{Md})$ & Variationsbreite $(\mathrm{R})$ & $\mathrm{H}_{\mathrm{u}}(\%)(<1,5 \mathrm{Punkte})$ & $\mathrm{H}_{\mathrm{o}}(\%)(>6,5$ Punkte $)$ \\
\hline Visuell erkannt & 186 & 2 & $7,5_{\max }-0_{\min }$ & 2 \\
Akustisch erkannt & 186 & 0 & $2{ }_{\max }-0_{\min }$ & 96 & 0 \\
\hline
\end{tabular}


Abb. 2 Prozentualer Anteil der im Test zur visuellen und akustischen Artenkenntnis von Berliner Schüler_innen erreichten Punkte beim Erkennen von acht einheimischen Vogelarten, $N=186$. Korrekter Artname: 1 Punkt, korrekte Gattung: 0,5 Punkte
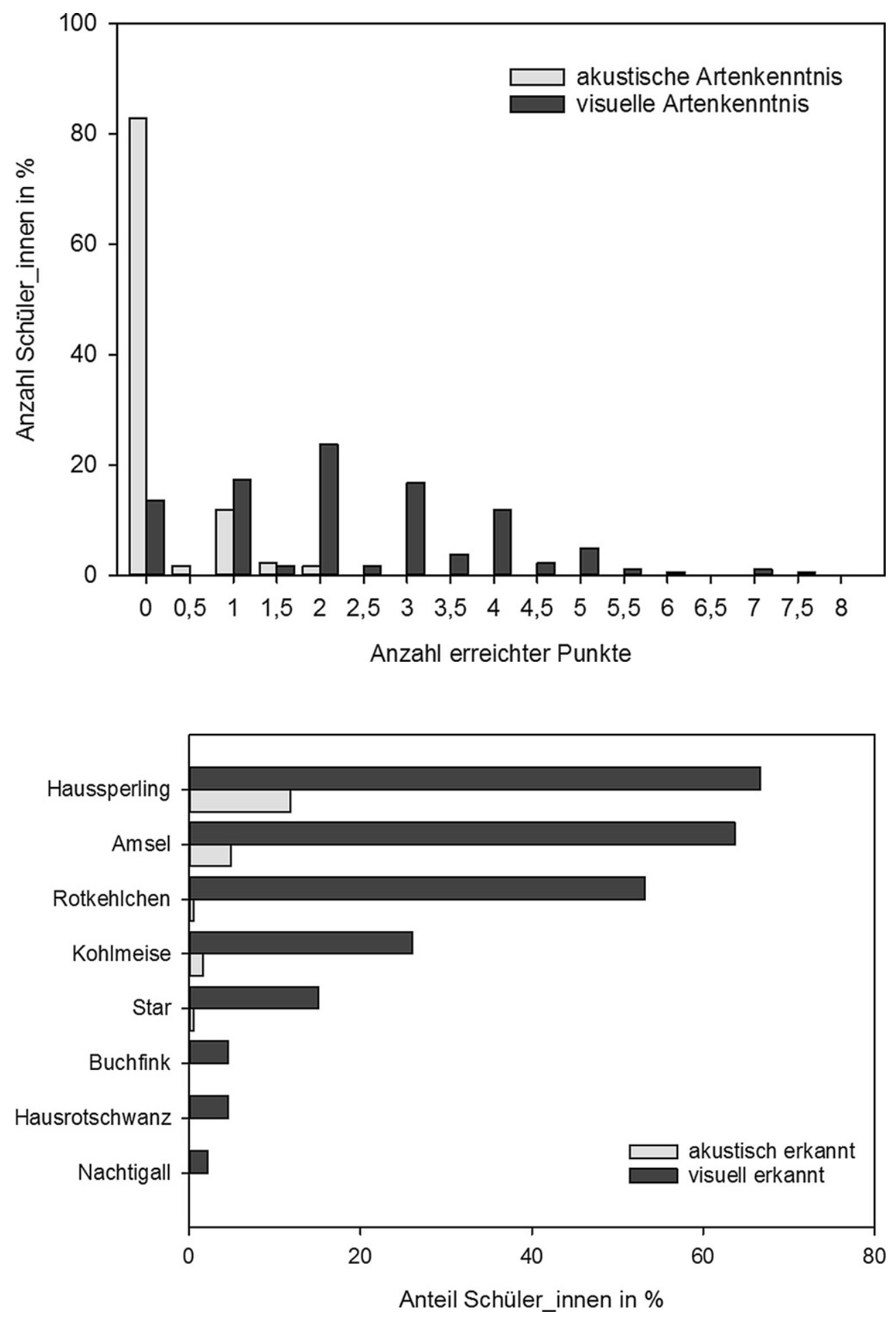

Abb. 3 Prozentualer Anteil der visuellen und akustischen Artenkenntnis von Berliner Schüler_innen, die eine Vogelart am Beispiel von acht einheimischen Vogelarten korrekt erkannten, $N=186$

\section{Ergebnisse}

\section{Phase 1 - Selbsteinschätzung der Artenkenntnis}

Die Schüler_innen schätzten ihre Fähigkeit einheimische Vogelarten anhand des Gesangs zu erkennen signifikant schlechter ein als ihre Fähigkeit diese Arten anhand vi- sueller Merkmale zu erkennen (Wilcoxon-Test: $N=149$, $Z=-9,5 ; p<0,001)$. Die Effektstärke entspricht einem starken Effekt (Korrelationskoeffizent: $r=-0,55$ ). Die Antworten der Selbsteinschätzung zeigen insgesamt eine geringe mittlere Artenkenntnis bei einer hohen Variationsbreite (Tab. 3). 
Weniger als 5\% der Schüler_innen glaubten, dass sie ausschließlich den Gesang einer Art erkennen können (Abb. 1). Eine Ausnahme stellte die Nachtigall dar. 12\% der Schüler_innen (18 Personen) gaben an, den Gesang der Nachtigall zu erkennen, sie jedoch nicht visuell identifizieren zu können.

\section{Phase 2 - tatsächliche Artenkenntnis}

Eine deutliche Mehrheit von 96\% der Schüler_innen, die an der Studie teilnahmen, hatten eine geringe akustische Artenkenntnis und $31 \%$ der Befragten eine geringe visuelle Artenkenntnis (Tab. 4). Insgesamt erkannten die befragten Schüler_innen signifikant weniger Vogelarten akustisch als visuell (Wilcoxon-Test: $N=186, \mathrm{Z}=-10,922 ; p<0,001$ ). Die Effektstärke ist stark $(r=-0,566)$. Die Ergebnisse zeigen eine leichte linksschiefe Verteilung für die visuelle Artenkenntnis und eine deutliche linksschiefe Verteilung für die akustische Artenkenntnis (Abb. 2).

Alle getesteten Vogelarten wurden häufiger visuell als akustisch erkannt (Abb. 3). Die Schüler_innen erkannten den Haussperling visuell und akustisch am häufigsten. In der Auswertung der Bezeichnung Spatz als Gattung war der Anteil der Schüler_innen, die ihn visuell erkannten jedoch geringer (38\%). Für die akustische Artenkenntnis wäre er weiterhin am häufigsten erkannt worden (6\%). Von den 22 Schüler_innen die eine Vogelart akustisch erkann- ten, erkannten 15 Schüler_innen diese Art auch visuell. Sieben Schüler_innen erkannten ausschließlich den Gesang einer Vogelart, konnten jedoch keine korrekte visuelle $\mathrm{Zu}$ ordnung derselben Art machen. Ein_e Schüler_in erkannte ausschließlich den Gesang der Kohlmeise, zwei Schüler_innen den Gesang der Amsel und vier der befragten Schüler_innen den Gesang des Haussperlings.

\section{Vergleich Selbsteinschätzung und Artenkenntnis}

Insgesamt schätzten die befragten Schüler_innen ihre Artenkenntnis über einheimische Vögel signifikant besser ein als sie tatsächlich in der Phase 2 war (Tab. 5). Die Anzahl, der von den Schüler_innen im Test erkannten Vogelarten lag sowohl visuell als auch akustisch unter dem selbst eingeschätzten Wert. Die Schüler_innen schätzten ihre Kenntnisse für alle Arten, mit Ausnahme des Hausrotschwanzes, höher ein als sie im Test waren (Abb. 4). Insbesondere die Kenntnis über die Nachtigall wurde von den Schüler_innen deutlich zu gut eingeschätzt.

\section{Verwechslungen}

Die Antworten der befragten Schüler_innen zeigten bestimmte Muster für visuelle Verwechslungen, die darauf schließen lassen, dass eine Zuordnung häufig basierend auf einer auffälligen Gefiederfarbe erfolgte (Abb. 5). Zudem

Tab. 5 Unterschied der visuellen und akustischen Artenkenntnis über Vögel (jeweils Median mit Variationsbreite) zwischen Selbsteinschätzung und Testleistung der befragten Berliner Schüler_innen für die acht getesteten Vogelarten, Wilcoxon-Test für abhängige Stichproben und Effektstärke r, $N=149$

\begin{tabular}{lllll}
\hline & Selbsteinschätzung & Testergebnis Md (R) & Wilcoxon-Test & Effektstärke r \\
& Md (R) & & $p$ & $-0,42$ \\
Visuelle Artenkenntnis & $4\left(8_{\max }-0_{\min }\right)$ & $2\left(7,5_{\max }-0_{\min }\right)$ & $<0,001$ & $<0,001$ \\
Akustische Artenkenntnis & $1\left(7_{\max }-0_{\min }\right)$ & $0\left(2_{\max }-0_{\min }\right)$ & $<, 39$ & -0, \\
\hline
\end{tabular}

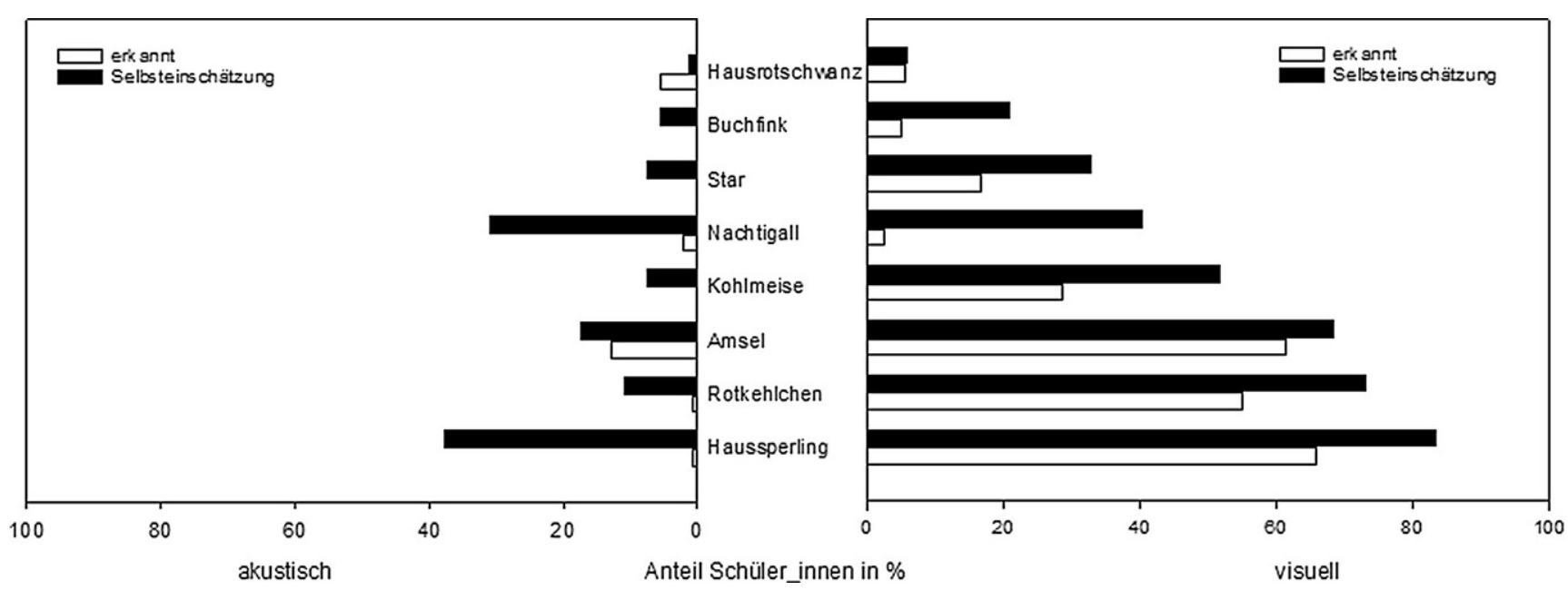

Abb. 4 Prozentualer Vergleich der Antworten der befragten Schüler_innen in Bezug auf ihre Selbsteinschätzung und das tatsächliche Erkennen für acht einheimische Vogelarten; $N=149$ 


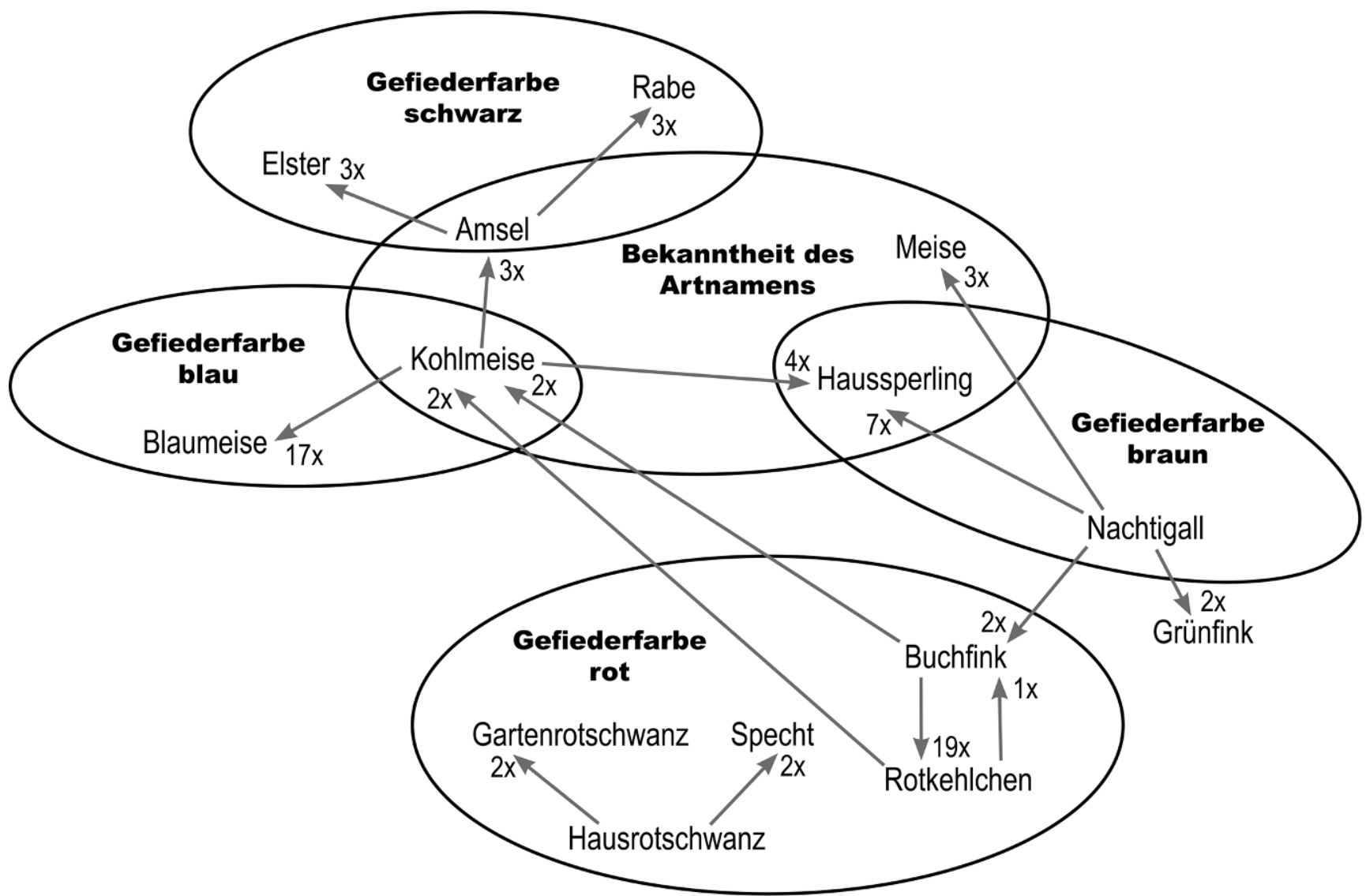

Abb. 5 Benennungen der Vogelarten im Test der visuellen Artenkenntnis. Pfeil „,benannt mit“

wurden Artnamen von Vögeln, die als bekannt einzuschätzen sind, häufig verwendet und falsch zugeordnet. Dieses Muster zeigten auch die falschen Zuordnungen des Gesangs. Haussperling (39-mal), Amsel (22-mal) und Nachtigall (10-mal) wurden am häufigsten als Artname falsch verwendet. Mehrere Schüler_innen verwechselten zudem den Gesang der Nachtigall mit dem des Spechts und den Gesang der Kohlmeise mit dem des Kuckucks.

\section{Diskussion}

Am Beispiel acht häufig vorkommender Brutvogelarten konnten wir zeigen, dass die derzeitige Artenkenntnis der befragten Berliner Schüler_innen der 7. Jahrgangsstufe eher gering ist. Die Schüler_innen erkannten im Mittel visuell nur zwei von acht Arten und damit weniger als ein Drittel der getesteten Vögel. Ferner wurden lediglich drei Vogelarten von mehr als der Hälfte der befragten Schüler_innen visuell erkannt: Haussperling, Amsel und Rotkehlchen.

Mit diesem Ergebnis liegt die Artenkenntnis der an der Studie teilnehmenden Berliner Schüler_innen unter der durchschnittlichen Kenntnis von Bayrischen Schüler_innen, die etwas mehr als ein Drittel der in der Studie von Gerl und Kolleg_innen (2018) getesteten, häufigen einheimischer Arten erkannten. Ein direkter Vergleich mit früheren Studien ist jedoch nicht möglich, da wir in unserer Studie mittels Videoaufnahmen testeten, während vorherige Studien Fotos (Randler 2006, 2008a; Gerl et al. 2018) oder Präparate (Zahner et al. 2007) verwendeten.

Ein Vergleich der visuellen Testergebnisse mit den Ergebnissen von Randler (2006) und Gerl und Kolleg_innen (2018) für einzelne Arten, die in allen drei Studien getestet wurden, zeigte sich die gleiche absteigende Reihenfolge in der Bekanntheit von Amsel, Rotkehlchen, Kohlmeise, Star und Buchfink. Die Berliner Schüler_innen erkannten diese Arten jedoch durchweg seltener als die befragten Schüler_innen bei Randler (2006) und Gerl und Kolleg_innen (2018). Eine Ausnahme stellte der Haussperling dar: Diese Art wurde bei Gerl et al. (2018) ebenfalls im Test eingesetzt und von den befragten Bayrischen Schüler_innen in der Reihenfolge seltener erkannt als die Kohlmeise. Die Berliner Schüler_innen erzielten bessere Testergebnisse in Bezug auf den Haussperling und er war die am häufigsten erkannte Vogelart. Allerdings unterscheidet sich die Auswertung unserer Ergebnisse zum Haussperling methodisch zur Auswertung von Gerl und Kolleg_innen (2018). Die Berliner Schüler_innen würden schlechtere Testergebnisse 
mit der Punktevergabe nach Gerl et al. (2018) erzielen. Dennoch wäre der Haussperling auch nach dieser Auswertung den Berliner Schüler_innen immer noch bekannter. Für eine genaue Einschätzung müssten aber die Ergebnisse zum Haussperling von Gerl und Kolleg_innen (2018) nach unserem Vorgehen ausgewertet und verglichen werden.

Der Haussperling war auch in der Selbsteinschätzung auf dem zweiten Platz der Arten, bei denen die Berliner Schüler_innen am häufigsten glaubten, sie erkennen zu können. Zudem wurden in der visuellen und akustischen Artenkenntnis die besten Testergebnisse erzielt. In Berlin gibt es aktuell eine große und stabile Population von Haussperlingen (Witt und Steiof 2013). Mit Ausnahme des sehr charakteristischen Rotkehlchens sind die Testergebnisse zum tatsächlichen Erkennen der anderen Vogelarten in etwa in der Reihenfolge ihrer Häufigkeit in Berlin (siehe Tab. 2). Dies deutet darauf hin, dass die Vogelarten, die die Schüler_innen oft beobachten oder die ihnen begegnen könnten, eher erkannt werden. Eine weitere Analyse der Anzahl der Meldungen von Beobachtungen von Vogelarten in $\mathrm{Ci}$ tizen Science Beobachtungsnetzwerken wie ornitho.de und naturgucker.de könnten weitere Einblicke geben, da neben der Anzahl an Individuen auch die Möglichkeit eine Art in Berlin zu beobachten berücksichtigt werden würde.

\section{Unterschied von akustischer und visueller Artenkenntnis}

Die Schüler_innen zeigten einen deutlichen Unterschied in ihrer visuellen und ihrer akustischen Artenkenntnis über einheimische Vögel. Insgesamt erzielten sie deutlich bessere Testleistungen bei der visuellen Artenkenntnis. Die akustische Artenkenntnis fällt vermutlich sogar noch geringer aus, da die Ergebnisse, wie wir annehmen, auch zufällig richtige Zuordnungen durch raten einschließen könnten. Ein Hinweis darauf ist, dass Vogelarten, die am häufigsten richtig erkannt wurden (Haussperling und Amsel), ebenfalls am häufigsten falsch genannt wurden. Schreck und Randler (2007) konnten ebenfalls eine nur geringe Kenntnis von Vogelstimmen bei Schüler_innen feststellen. In ihrer Studie wiesen sie auf die Schwierigkeiten der Vermittlung von akustischer Artenkenntnis hin. Zudem werden Jugendliche vermutlich eher mit Bildern und den begleitenden Artbezeichnungen z.B. in Büchern konfrontiert, als dass sie aktiv auf unterschiedliche Vogelstimmen aufmerksam gemacht werden. Wir nehmen an, dass Mentor_innen und persönliche Naturerfahrung für die akustische Artenkenntnis eine noch entscheidendere Rolle spielen als für die visuelle Artenkenntnis von Vögeln.

\section{Verwechslungen}

Die in unserer Studie herausgearbeiteten Verwechslungen deuten darauf hin, dass für die Zuordnung von den Schüler_innen ähnlich wie in der Studie von Hesse (2002) besonders auffällige Merkmale isoliert genutzt wurden. Auch wurden oft allgemein bekannte und vertraute Artnamen (z.B. Spatz und Amsel) verwendet, was möglicherweise zu Verwechslungen führte. Wir konnten ebenfalls wie Gerl und Kolleg_innen (2018) eine Verbindung zwischen auffälligen Gefiederfarben und Namen wie zum Beispiel Rotkehlchen, die auf Merkmale hinweisen, finden. In der akustischen $\mathrm{Zu}$ ordnung könnte der Rhythmus des Gesangs von den Schüler_innen in der Zuordnung als Merkmal verwendet worden sein. Für eine bessere Interpretation der Ergebnisse wäre es jedoch förderlich, in zukünftigen Forschungsarbeiten zu untersuchen, welche Bestimmungsmerkmale Schüler_innen kennen und bewusst als Strategie zur Arterkennung verwenden.

\section{Selbsteinschätzung}

Übereinstimmend mit den Ergebnissen von Hesse (2002) schätzten die an unserer Studie teilnehmenden Schüler_innen ihre Artenkenntnis über einheimische Vögel deutlich besser ein als sie tatsächlich war. Jedoch zeigte sich, dass die Schüler_innen in der Einschätzung ihrer Unkenntnis zuverlässige Aussagen machten. Die Schüler_innen gaben bei ihrer Selbsteinschätzung die niedrigsten Werte für den Hausrotschwanz an. Diese stimmten mit den Testergebnissen der tatsächlichen visuellen Artenkenntnis überein. Mit dieser Selbsteinschätzung der Wissenslücken besteht eine wichtige Grundlage für das Bewusstsein eines Bedarfs der Erweiterung ihrer Kenntnisse (Boud und Flachikov 1989).

Wir schlussfolgern, dass die Kenntnis und Vertrautheit mit den Artnamen dabei ein wichtiges Kriterium für die Selbsteinschätzung der an unserer Studie teilnehmenden Schüler_innen waren. Beispielsweise schätzten die Schüler_innen die Erkennung der Nachtigall, insbesondere des Gesangs, vorab als gut ein. Diese Einschätzung erklären wir uns damit, dass die Nachtigall sowie ihr außergewöhnlich vielseitiger Gesang, häufig in Literatur und Popkultur erwähnt wird und der Artname den Schüler_innen daher vertraut schien. Diese Beobachtung unterstützt die Ergebnisse von Hesse (2002), die zeigten, dass eine reine Namensabfrage nicht die tatsächliche Artenkenntnis wiedergibt, sondern lediglich das Kennen des Artnamens abfragt. 


\section{Bedeutung der Ergebnisse für die Entwicklung von Lernangeboten}

Aus den Ergebnissen unserer Studie schlussfolgern wir, dass in Berlin ein erhöhter Bedarf an Lernangeboten zur Förderung der Artenkenntnis für Schüler_innen besteht. Dies hat eine große Tragweite im Kontext der Biodiversitätskrise, da die Vermittlung des Verlusts von Biodiversität in Bezug auf die persönliche Umgebung in Berlin (van Weelie und Wals 2010) derzeit kaum an Vorwissen anknüpfen kann.

Darüber hinaus zeigen unsere Ergebnisse, wie wichtig es ist, den kulturellen Aspekt von Artnamen und den damit verbundenen Vorstellungen der Schüler_innen bei der Vermittlung von Artenvielfalt mit einzubeziehen. Der konstruktivistischen Auffassung von Lernen nach ReinmannRothmeier und Mandl (2001) folgend ist der Erwerb von Artenkenntnis ein aktiver Konstruktionsprozess, in dem jede_r Lernende abhängig von Vorwissen, Einstellungen und Lernsituation Wissen aktiv neu konstruiert. Mayer und Horn (1993) betonen die Bedeutung unterschiedlicher kulturell geprägter Begegnungsweisen mit Organismen. Artnamen beschreiben nicht nur häufig charakteristische Merkmale und lösen dadurch bestimmte Erwartungen aus (Gerl et al. 2018), sondern sind häufig mit kulturellen Wahrnehmungen verbunden.

Generell erscheint der Erwerb von Artenkenntnis in Verbindung mit einer direkten Naturbeobachtung am wirksamsten (Mayer und Horn 1993; Randler und Bogner 2002; Gerl et al. 2018; Schulte et al. 2019). Es ist dabei jedoch wichtig, Schüler_innen nicht mit einer zu großen Vielzahl an Arten und veränderten Lernsituation zu überfordern (Eschenhagen 1985; Schreck und Randler 2007; Randler 2008b). Oftmals sind direkte Naturerfahrungen im Schulalltag aufgrund verschiedener Faktoren (wie z. B. Zeitmangel, Mangel an Betreuungspersonen, Curricula) schwer zu vereinbaren (Kelemen-Finan und Dedova 2014; Gebhard und Menzel 2019). In diesem Zusammenhang kann der Schulgarten bzw. das Schulgelände eine Alternative für Exkursionen sein und bietet als eine von vielen didaktischen Funktionen (Retzlaff-Fürst 2013) die Möglichkeit, gezielt für die Förderung von Artenkenntnis gestaltet zu werden (Eschenhagen 1985). Randler (2008b) zeigte, dass sich auch Berichte von Mitschüler_innen über Naturerfahrungen positiv auf den Wissenszuwachs von Schüler_innen auswirken können. Dem folgend empfahl er Exkursionen in den Erfahrungsraum der Schüler_innen in wechselnden Gruppen nach einer angemessenen Vorbereitung im Klassenzimmer (Randler und Bogner 2002; Randler 2008b).

Es gibt bereits Programme, die für die Praxis der Förderung von Artenkenntnis durch die Lehrkräfte sehr geeignet erscheinen, wie z.B. die Fünf-Minuten Biologie (Eschenhagen 1985; Mayer und Horn 1993; Mohr et al. 2018).
Beim Konzept der Fünf-Minuten-Biologie werden unabhängig vom anstehenden Unterrichtsstoff aktuelle Inhalte der Lebens- und Erlebniswelt der Schüler_innen im Unterricht in begrenztem Zeitumfang von ca. fünf Minuten spontan behandelt (Stichmann 1992). Es sollte jedoch dabei nicht außer Acht gelassen werden, dass die Lehrpersonen oftmals selbst über eine geringe Artenkenntnis verfügen (Kelemen-Finan und Dedova 2014). Für eine tiefgehende und praktische Förderung von Artenkenntnis müssen daher auch im schulischen Umfeld wieder mehr Mentor_innen ausgebildet werden.

Ebenfalls ist es wichtig zu beachten, dass das Erlernen von Artenkenntnis ein Selbstlernprozess ist, der zwar in der Schule angeregt werden kann, aber meist darüber hinaus (intrinsischer) Motivation und Interesse bedarf (Schulte et al. 2019). Der Lernprozess sollte daher aktiv, selbstgesteuert, sozial, situativ und konstruktiv gestaltet sein (Reinmann-Rothmeier und Mandl 2001) und z. B. durch regelmäBige Besuche von außerschulischen Lernorten unterstützt werden (Schulte et al. 2019). Schneider und Wildt (2009) betonen, dass beim forschenden Lernen der Forschungsprozess durch Verknüpfung mit dem Lernprozess bedeutsame Erkenntnisse für die Lernenden erzeugt. Die Partizipation an wissenschaftlichen Projekten (Citizen Science) kann demnach zu einem positiven Effekt auf das Interesse und die motivationale Regulation von Schüler_innen führen (Hiller und Kitsantas 2014; Silva et al. 2016; White et al. 2018).

\section{Ausblick}

Um dem Verlust der Artenvielfalt mit Artenkennern von morgen entgegen zu wirken, sollte in Berlin ein umfassendes und strukturiertes Programm von Lernangeboten für den Erwerb und die Förderung von Artenkenntnis aufgebaut werden. Ein solches Programm sollte insbesondere Schulen aber auch außerschulische Lernorte in ihrer Arbeit unterstützen. Beispielsweise könnten Lehrkräfte mit einer Übersicht von Arten und Kontexten, die sich für die Förderung von Artenkenntnis besonders eignen, unterstützt werden. Zudem sollten Methoden entwickelt und getestet werden, wie das Interesse der Schüler_innen individuell erfasst und in die Lernangebote besser integriert werden kann.

Besonders interessant vor dem Hintergrund der Ergebnisse dieser Studie in Bezug auf die Nachtigall wäre es, den Effekt des Citizen Science Projekts Forschungsfall Nachtigall auf das Interesse und die Artenkenntnis von Schüler_innen zu untersuchen. Dieses Projekt nutzt die in der Kultur fest verankerte Bekanntheit dieses charismatischen Vogels, verknüpft kulturelle mit biologischen Aspekten und erforscht gemeinsam mit Schüler_innen die geographischen Gesangsmuster von Nachtigallen in der Stadtnatur Berlins. 
Danksagung Wir danken Sarah Darwin, Madeleine Hallmann, Anke Hoffmann, Susan Karlebowski, Nathalie Köppe und Susan Scheyda für ihre Unterstützung bei der Befragung der Schüler_innen. Wir bedanken uns bei den beteiligten Schulen, Lehrkräften und Schüler_innen für ihre Teilnahme und Unterstützung.

Förderung Diese Publikation entstand im Rahmen der Projekte Stadtnatur entdecken, gefördert durch das Bundesministerium für Umwelt, Naturschutz und nukleare Sicherheit (BMU) und Forschungsfall Nachtigall, gefördert durch das Bundesministerium für Bildung und Forschung (BMBF).

Funding Open Access funding provided by Projekt DEAL.

Open Access Dieser Artikel wird unter der Creative Commons Namensnennung 4.0 International Lizenz veröffentlicht, welche die Nutzung, Vervielfältigung, Bearbeitung, Verbreitung und Wiedergabe in jeglichem Medium und Format erlaubt, sofern Sie den/die ursprünglichen Autor(en) und die Quelle ordnungsgemäß nennen, einen Link zur Creative Commons Lizenz beifügen und angeben, ob Änderungen vorgenommen wurden.

Die in diesem Artikel enthaltenen Bilder und sonstiges Drittmaterial unterliegen ebenfalls der genannten Creative Commons Lizenz, sofern sich aus der Abbildungslegende nichts anderes ergibt. Sofern das betreffende Material nicht unter der genannten Creative Commons Lizenz steht und die betreffende Handlung nicht nach gesetzlichen Vorschriften erlaubt ist, ist für die oben aufgeführten Weiterverwendungen des Materials die Einwilligung des jeweiligen Rechteinhabers einzuholen.

Weitere Details zur Lizenz entnehmen Sie bitte der Lizenzinformation auf http://creativecommons.org/licenses/by/4.0/deed.de.

\section{Literatur}

Bergmann, H.H. (2017). Vogelstimmen. Wie man sie kennenlernt und was man an ihnen entdecken kann. Biologie in unserer Zeit, 3(47), 178-185. https://doi.org/10.1002/biuz.201710621.

Boud, D., \& Flachikov, N. (1989). Quantitative studies of student selfassessment in higher education: a critical analysis of findings. Higher Education, 15(3), 529-549.

Brämer, R., Koll, H., \& Schild, H.-J. (2016). Natur Nebensache? 7. Jugendreport Natur 2016. Erste Ergebnisse. Köln: Universität Köln.

Buck, T., Bruchmann, I., Zumstein, P., \& Drees, C. (2019). Just a small bunch of flowers: the botanical knowledge of students and the positive effects of courses in plant identification at German universities. PeerJ. https://doi.org/10.7717/peerj.6581.

Cox, D.T.C., \& Gaston, K. J. (2015). Likeability of garden birds: importance of species knowledge \& richness in connecting people to nature. PLOS ONE. https://doi.org/10.1371/journal.pone. 0141505 .

Dallimer, M., Irvine, K. N., Skinner, A. M. J., Davies, Z. G., Rouquette, J.R., Maltby, L.L., et al. (2012). Biodiversity and the feel-good factor: understanding associations between self-reported human well-being and species richness. Bioscience, 62(1), 47-55. https:// doi.org/10.1525/bio.2012.62.1.9

Díaz, S., Settele, J., Brondizio, E.S., Ngo, H. T., Guèze, M., Agard, J., et al. (Hrsg.). (2019). Summary for policymakers of the global assessment report on biodiversity and ecosystem services of the Intergovernmental Science-Policy Platform on Biodiversity and Ecosystem Services. Bonn: IPBES.

Eschenhagen, D. (1985). Vermittlung von Pflanzen- und Tierkenntnissen in der Grundschule. Sachunterricht und Mathematik in der Primarstufe, 13(4), 120-126.

Frobel, K., \& Schlumprecht, H. (2016). Erosion der Artenkenner. $\mathrm{Na}$ turschutz und Landschaftsplanung, 48(4), 105-113.
Gebhard, U., \& Menzel, S. (2019). Naturwahrnehmung von Kindern und Jugendlichen. In J. Groß, M. Hammann, P. Schmiemann \& J. Zabel (Hrsg.), Biologiedidaktische Forschung: Erträge für die Praxis (S. 269-285). Berlin, Heidelberg: Springer Spektrum.

Gerl, T., Almer, J., Zahner, V., \& Neuhaus, B. J. (2018). Der BISATest: Ermittlung der Formenkenntnis von Schülern am Beispiel einheimischer Vogelarten. Zeitschrift für Didaktik der Naturwissenschaften, 24(1), 235-249.

Hesse, M. (2002). Eine neue Methode zur Überprüfung von Artenkenntnissen bei Schülern; Frühblüher: Benennen, Selbsteinschätzen, Wiedererkennen. Zeitschrift für Didaktik der Naturwissenschaften, 8, 53-66.

Hiller, S.E., \& Kitsantas, A. (2014). The effect of a horseshoe crab citizen science program on middle school student science performance and STEM career motivation. School Science and Mathematics. https://doi.org/10.1111/ssm.12081.

Hooykaas, M. J.D., Schilthuizen, M., Aten, C., Hemelaar, E. M., Albers, C. J., \& Smeets, I. (2019). Identification skills in biodiversity professionals and laypeople: a gap in species literacy. Biological Conservation. https://doi.org/10.1016/j.biocon.2019.108202.

Jäkel, L., \& Schaer, A. (2004). Sind Namen nur Schall und Rauch? Wie sicher sind Pflanzenkenntnisse von Schülerinnen und Schülern? Berichte des Institutes für Didaktik der Biologie, 13, 1-24.

Kattmann, U., Duit, R., Gropengießer, H., \& Komorek, M. (1997). Das Modell der Didaktischen Re-konstruktion - Ein Rahmen für naturwissenschaftsdidaktische Forschung und Entwicklung. Zeitschrift für Didaktik der Naturwissenschaften, 3(3), 3-18.

Kelemen-Finan, J., \& Dedova, I. (2014). Vermittlung von Artenkenntnis im Schulunterricht. Naturschutz und Landschaftsplanung, 46(7), 219-225.

KMK [Sekretariat der Ständigen Konferenz der Kultusminister der Länder] (2004). Bildungsstandards im Fach Biologie für den Mittleren Schulabschluss. München: Wolters Kluwer.

Krüger, D., \& Burmester, A. (2005). Wie Schüler Pflanzen ordnen. Zeitschrift für Didaktik der Naturwissenschaften, 11, 85-102.

Maher, J.M., Markey, J.C., \& Ebert-May, D. (2013). The other half of the story: effect size analysis in quantitative research. $C B E$ life sciences education, 12(3), 345-351. https://doi.org/10.1187/cbe. 13-04-0082.

Mayer, J. (1992). Formenvielfalt im Biologieunterricht. Ein Vorschlag zur Neubewertung der Formenkunde. Kiel: IPN.

Mayer, J., \& Horn, F. (1993). Formenkenntnis - wozu? Unterricht Biologie, 17(189), 4-13.

Mohr, K., Vermaseren, P., Hense, J., \& Scheersoi, A. (2018). Apfel, Birke, Purzelbaum? Förderung von Artenkenntnis und Interesse an Bäumen mit Hilfe der 5-Minuten-Biologie. In 200 Jahre Rheinische Friedrich-Wilhelms-Universität Bonn. Bonn. Vortrag.

Randler, C. (2003). Amsel, Drossel, Fink und Star ... Welche Vogelarten kennen Schülerinnen und Schüler? Praxis der Naturwissenschaften - Biologie in der Schule, 52(1), 44-45.

Randler, C. (2006). War früher alles besser? Eine Untersuchung zu Wirbeltierartenkenntnissen bei Schülerinnen und Schülern. Natur und Landschaft, 36, 181-188.

Randler, C. (2008a). Pupils' factual knowledge about vertebrate species. Journal of Baltic Science Education, 7(2), 48-54.

Randler, C. (2008b). Teaching species identification-A prerequisite for learning biodiversity and understanding ecology. Eurasia Journal of Mathematics, Science \& Technology Education, 4(3), 223-231.

Randler, C., \& Bogner, F. (2002). Comparing methods of instruction using bird species identification skills as indicators. Journal of Biological Education, 36, 181-188.

Randler, C., \& Wieland, L. (2010). Knowledge about common vertebrate species in German kindergarten pupils. Journal of Baltic Science Education, 9, 135-141.

Reinmann-Rothmeier, G., \& Mandl, H. (2001). Unterrichten und Lernumgebungen gestalten. In A. Krapp \& B. Weidenmann (Hrsg.), 
Pädagogische Psychologie. Ein Lehrbuch (S. 601-646). Weinheim: Beltz.

Remmele, M., \& Lindemann-Matthies, P. (2018). Like father, like son? On the relationship between parents' and children's familiarity with species and sources of knowledge about plants and animals. EURASIA Journal of Mathematics, Science and Technology Education. https://doi.org/10.29333/ejmste/92287.

Retzlaff-Fürst, C. (2013). Schulgelände und Schulgarten. In H. Gropengießer, U. Harms \& U. Kattmann (Hrsg.), Fachdidaktik Biologie (S. 421-428). Hallbergmoos: Aulis.

Rieckmann, M., Mindt, L., \& Gardiner, S. (2017). Education for sustainable development goals. Learning objectives. Paris, France: United Nations Educational, Scientific and Cultural Organization.

Schneider, R., \& Wildt, J. (2009). Forschendes Lernen und Kompetenzentwicklung. In L. Huber, J. Hellmer \& F. Schneider (Hrsg.), Forschendes Lernen im Studium. Aktuelle Konzepte und Erfahrungen (S. 53-68). Bielefeld: Universitätsverlag Webler.

Schreck, S., \& Randler, C. (2007). Vogelstimmen lernen - eine Evaluation zweier Unterrichtskonzepte. Der mathematische und naturwissenschaftliche Unterricht, 60(6), 362-365.

Schulte, R., Jedicke, E., Lüder, R., Linnemann, B., Munzinger, S., von Ruschkowski, E., et al. (2019). Eine Strategie zur Förderung der Artenkenntnis. Naturschutz und Landschaftsplanung, 51(5), 210-217.

Senatsverwaltung für Bildung Jugend und Sport Berlin (2006). Naturwissenschaften. Rahmenlehrplan Grundschule. Berlin: Wissenschaft und Technik.

Silva, C. G., Monteiro, A., Manahl, C., Lostal, E., Holocher-Ertl, T., Andrade, N., et al. (2016). Cell spotting: educational and motivational outcomes of cell biology citizen science project in the classroom. Journal of Science Communication, 15(1), A2.

Steffen, W., Richardson, K., Rockstrom, J., Cornell, S.E., Fetzer, I., Bennett, E. M., et al. (2015). Planetary boundaries: guiding human development on a changing planet. Science. https://doi.org/ 10.1126/science. 1259855 .
Stichmann, W. (1992). Das Konzept der Fünf-Minuten-Biologie. Unterricht Biologie, 176, 4-13.

Sturm, P., \& Berthold, T. (2015). Biodiversität im Unterricht - ein Konzept zur Umsetzung der Bayerischen Biodiversitätsstrategie im schulischen Bereich. Anliegen Natur, 37(2), 76-83.

Svensson, L., Grant, P. J., Mullarny, K., \& Zetterström, D. (1999). Der neue Kosmos Vogelführer. Alle Arten Europas, Nordafrikas und Vorderasiens. Stuttgart: Franckh-Kosmos.

Tancoigne, E., Bole, C., Sigogneau, A., \& Dubois, A. (2011). Insights from zootaxa on potential trends in zoological taxonomic activity. Frontiers in Zoology. https://doi.org/10.1186/1742-9994-8-5.

Tiemann, R., \& Körbs, C. (2014). Die Fragebogenmethode, ein Klassiker der empiri-schen didaktischen Forschung. In D. Krüger, I. Parchmann \& H. Schecker (Hrsg.), Methoden in der naturwissenschaftsdidaktischen Forschung (S. 283-295). Berlin: Springer Spektrum.

Wägele, H., Klussmann-Kolb, A., Kuhlmann, M., Haszprunar, G., Lindberg, D., Koch, A., et al. (2011). The taxonomist-an endangered race. A practical proposal for its survival. Frontiers in Zoology. https://doi.org/10.1186/1742-9994-8-25.

van Weelie, D., \& Wals, A. (2010). Making biodiversity meaningful through environmental education. International Journal of Science Education, 24(11), 1143-1156. https://doi.org/10.1080/ 09500690210134839.

White, R.L., Eberstein, K., \& Scott, D. M. (2018). Birds in the playground: evaluating the effectiveness of an urban environmental education project in enhancing school children's awareness, knowledge and attitudes towards local wildlife. PLOS ONE. https://doi.org/10.1371/journal.pone.0193993.

Witt, K., \& Steiof, K. (2013). Rote Liste und Liste der Brutvögel von Berlin. 3. Fassung. 15. 11. 2013. Berliner orthologischer Bericht, $23,1-23$.

Zahner, V., Blaschke, S., Fehr, P., Herlein, S., Krause, K., Lang, B., et al. (2007). Vogelarten-Kenntnis von Schülern in Bayern. Vogelwelt, 128, 203-214. 